\title{
Data driven automatic model selection and parameter adaptation - a case study for septic shock
}

\author{
R. Brause \\ J.W.G.University, Frankfurt, Germany \\ RBrause@informatik.uni-frankfurt.de
}

\begin{abstract}
In bioinformatics, biochemical pathways can be modeled by many dfferential equations. It is still an open problem how to fit the huge amount of parameters of the equations to the available data. Here, the approach of systematically learning the parameters is necessary.

This paper propose as model selection criterion the least complex description of the observed data by the model, the minimum description length. For the small, but important example of inflammation modeling the performance of the approach is evaluated.
\end{abstract}

\section{Introduction}

In living organisms many metabolisms and immune reactions depend on specific, location-dependent interactions. Since the interactions occur in a timed transport of matter and molecules, this can be termed as a network of biochemical pathways of molecules. In Bioinformatics, these pathways or signal interactions are modeled by many differential equations. For complicated systems, differential equations systems (DES) with up to 7,000 equations and 20,000 associated parameters exist and model reality. The motivation for life science industry to use such systems is evident: A prediction of reactions and influences by simulated models helps avoiding timeconsuming, expensive animal and laboratory experiments, decrease the high costs for developing new drugs and therefore may save millions of Euros. For small signal transduction networks, this has already been done by estimating the parameters by data-driven modeling of expression profiles of DNA micro arrays, see e.g.[2]. Interestingly, no problems were reported fitting the models to the data.

Although the basic idea is quite seducing, the practical problems associated with the simulation approach are difficult to solve: How do we know that our selected model is valid and how can all parameters be set to the correct values? And if all parameters are different for each individual, how can they be adapted to the real values based only on a small set of measured data per organism?

In this paper we will try to answer some of theses questions for the example of the small but important problem of inflammation and septic shock.

\section{The differential equation neural network of inflammation and septic shock}

The symptoms of septic shock contain low blood pressure, high ventilation and high heart rates and may occur after an infection or a trauma (damage of tissue). The septic shock research has no convincing results yet; there is still a high mortality of about $50 \%$ on the intensive care units (ICU) and nobody knows why. It is only possible to predict the outcome for a patient in advance just for 3 days [1]. In 1999, about 250,000 death were associated with sepsis in the USA.

The septic shock state is produced by a confusing myriad of immune pathways and molecules. For studying the basic problems we restrict ourselves first to a simplified but still functional version of the model which uses only three variables and 12 constant parameters [3]. Let $P$ be the pathogen influence, $M$ the immunological response, e.g. the macrophages involved and $D$ the obtained cell damage. Then, using some basic assumptions [4], we might combine them into a coupled system of three first order differential equations (DES):

$$
\begin{aligned}
& P^{\prime}(t)=a_{1}(1-P) P+a_{2} M P \\
& M^{\prime}(t)=a_{3} M+a_{4} M(1-M) P+a_{5} M(1-M) D \\
& D^{\prime}(t)=a_{6} D+a_{7} h\left(\left(M-a_{9}\right) / a_{8}\right)
\end{aligned}
$$

The plot of the time course for the three outputs (three variables) for the set of parameters shown in Tab. 1 is shown in Fig. 1. For this, the differential equations were numerically integrated using the Runge-Kutta method.

$$
\begin{array}{lll}
\mathrm{a}_{1}=0.054 & \mathrm{a}_{3}=-1.0 & \mathrm{a}_{6}=-0.01 \\
\mathrm{a}_{2}=-0.2155 & \mathrm{a}_{4}=5.0 & \mathrm{a}_{7}=0.00384 \\
\mathrm{a}_{9}=0.2018 & \mathrm{a}_{5}=1.0 & \mathrm{a}_{8}=0.1644
\end{array}
$$

\section{Tab. 1 The constant parameter values}

It can be concluded that an infection (P) causes cell damage (D) and a delayed activity of the macrophages (M). The infection is defeated by the macrophages which decrease to a sufficient level afterwards. 


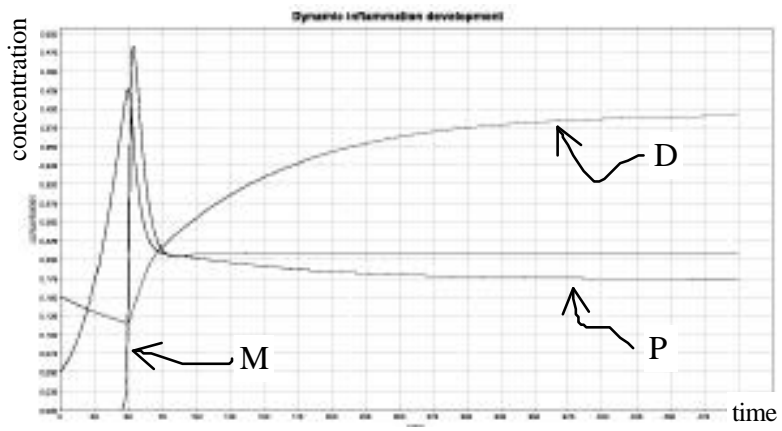

Fig. 1 The time dynamics of the equations (4), (5) and (6).

In this case (parameter regime), the infection remains chronically and the cell damage reaches a stable level. In the next section let us regard the adaptation of the parameters more closely.

\section{Learning the Parameters}

Generally, the biochemical pathways are very complex. It is not clear, which influences are important and which are not important. For the analytical description by equations this means that the number of terms ("model selection") and the values of its parameters ("model adaptation") are not given a priori, but have to be estimated ("learned") by the real observed data. How can this be done?

First, we are troubled by the fact that we do not have the full data set of Fig. 1 but only the small set of observed data. An example is given in table 2. For instance, the dynamics of inflammation might be in the range of hours, whereas the observed data is taken once each day.

\begin{tabular}{c|c|c|c}
$\begin{array}{c}\text { Time } \\
\text { step }\end{array}$ & $\mathbf{P}$ & $\mathbf{M}$ & $\mathbf{D}$ \\
\hline 0 & 0.05000 & 0.00100 & 0.15000 \\
& 0 & 0 & 0 \\
100 & 0.20121 & 0.20607 & 0.25434 \\
& 5 & 9 & 7 \\
200 & 0.18375 & 0.20684 & 0.34202 \\
& 1 & 4 & 7 \\
300 & 0.17727 & 0.20675 & 0.37428 \\
400 & 0 & 0 & 2 \\
& 0.17487 & 0.20668 & 0.38614 \\
500 & 6 & 0 & 1 \\
& 0.17399 & 0.20664 & 0.39050 \\
& 5 & 9 & 0
\end{tabular}

Tab. 2 The observed sparse data

In Fig. 2 this situation is shown. Here, the variable $y(t)$ changes after each time tick, but it is only measured at time points $t_{i}$. The different time scales will change heavily the approximated coefficients and difference equations, see [4]. Therefore, if we ignore the time steps between the observations and assume that system iterates once for one observation we will not be able to predict the best fitting parameters a for the difference equations that have several time steps between the observations.

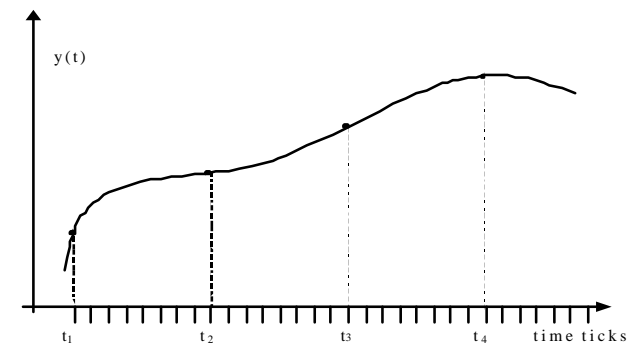

Fig. 2 The different time intervals for the differe $n$ tial equation and the observations

It turned out [4] that a variant of the classical evolutionary approach [5] did the job quite well. The advantage of this approach is its independency of the complexity of the objective function. The disadvantage is its high computational burden: we have to recompute the objective function each time we change only one parameter, and we can not adapt the step width in advance. Nevertheless, for a given DES and given observed data this approach shows good performance, see [4].

For a given model, this is fine. If the model is not given we are in trouble: How should we select the model and adapt the parameters at the same time? The initial idea of first adapting the parameters and then selecting the model by pruning all terms that has very small parameter values might work. Consider for instance our model of eqs. (1),(2),(3). We might consider the following ideas:

(1) If the pathogen influence (microbes) is present at the location where cell damage occurs, the pathogen influence will be increased: P' $\sim$ PD

(2) Macrophages will die due to toxic influence of mcrobes, proportional to the co-occurrence probability and the microbe concentration : $\mathrm{M}^{\prime} \sim-\mathrm{P}^{2} \mathrm{M}$

These two possible extensions of the model are translated into the modified differential equations

$$
\begin{aligned}
& P^{\prime}(t)=a_{1}(1-P) P+a_{2} M P+a_{10} P D \\
& M^{\prime}(t)=a_{3} M+a_{4} M(1-M) P+a_{5} M(1-M) D+a_{11} P^{2} M \\
& D^{\prime}(t)=a_{6} D+a_{7} h\left(\left(M-a_{9}\right) / a_{8}\right)
\end{aligned}
$$

On the other hand, we might have a more simple model in reality than we expect. For instance, we might have a 
model without influence of variable $D$ to variable $M$, i.e. $a_{5}$ $=0$, or a changed model with both $\mathrm{a}_{10}, \mathrm{a}_{11} \neq 0$ and $\mathrm{a}_{5}=0$.

With these ideas, we have now four different possible models. How can we decide which model is implemented by reality? How can we choose the best model?

\section{Model selection}

The choice of the model is important for all diagnosis and therapies of the septic process. First, we have to discuss several possibilities for selecting the appropriate model and then we will select one strategy of our choice.

\subsection{Model selection by parameter pruning}

As the first, naïve approach let us consider the case where we have the pure differential equations (1),(2),(3) or (4),(5),(6) encountering no nois e and we have recorded observation samples. How do we know which model is the right one for the observations? In this case, we might expect that the additional terms produce an error in mo deling the observations. In the other way, reducing the error in the parameter adaptation process might result in setting the unnecessary parameters to zero, if they exist: the describing model is automatically tailored to the observed data.

We observe that in the long run the approximation with additional terms does not improve while the correct model does. How can this be explained? The additional interactions that are caused by the additional parameters $\mathrm{a}_{10}$ and $\mathrm{a}_{11}$ in the augmented model will produce small disturbances that will deviate the approximation process: the approximation will slow down in relation to the nonaugmented model which fits well to the observed data. Also, adapt the parameters of all models.

So, another plausible idea might be: Select the model, which converges best. Is this a good selection criterion for the model? The answer is no: the additional interactions slow the convergence down, but the inverse is also true for too simple models which can not approximate the observed samples well, but initially converge faster than the true model.

Additionally, in nearly almost all natural systems we encounter noise that is not considered in this approach. So, we have to relay on other approaches.

\subsection{Model selection by minimum description length}

In the previous section we have seen that the convergence of the parameters cannot automatically replace the model selection process. Instead, we have to evaluate the performance of each model, i.e. each form of DES sepa- rately related to the observed time course samples. What kind of performance measure should we choose? We know that the deviation of the samples to the predicted values, the mean squared error, is not a good approach: by the additional parameters the more complex models will tend to overfit on adapting to the observed values perfectly whereas the best model will produce sample differences within the variance of the samples. This leads to our

Strategy: Adapt the parameters of all models to fit the observed data. Select the model which gives the shortest description of the observed data, on average and asymptotically.

So, we are looking for a model which neither fits too good nor too bad and needs only a small amount of information to describe the observations. How do we evaluate this?

Let us formalize our problem: For each of the $k$ subjects and each variable, we observe values at different time steps $t_{1}, t_{2}, \ldots, t_{n}$. For example as shown in Fig. 3, we might measure the dynamics at four times. All the four samples of one subject might be grouped together in one set. The set of observations for one subject is called a sample $\mathbf{x}=$ $\left(\mathrm{x}_{1}, \ldots, \mathrm{x}_{\mathrm{n}}\right)$ of all possible observations $\{\mathbf{x}\}$. Each model $\mathrm{m}$ which has been fit to the sample also produces by simulation a sample $\mathbf{f}=\left(\mathrm{f}_{1}, \ldots, \mathrm{f}_{\mathrm{n}}\right)$ for the designated $n$ time steps

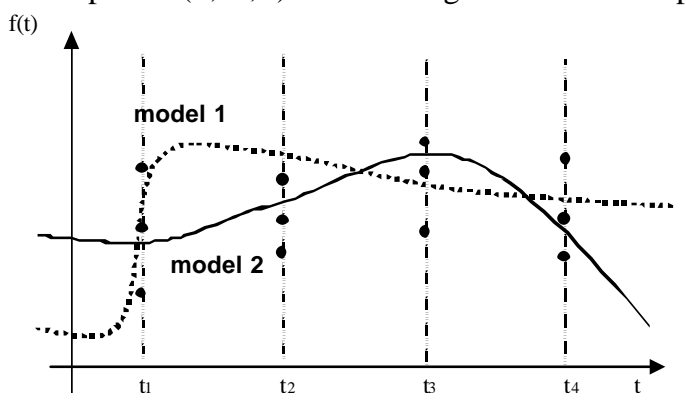

Fig. 3 Selecting the best fitting model

The deviation of the ith observed sample $\mathbf{x}(\mathrm{i})$ from its adapted model $\mathbf{f}(\mathrm{i})$ of the same type is for all time steps $\mathrm{t}=$ $1 \ldots N$ its empirical variance [6]

$$
\sigma_{i}^{2}=\frac{1}{N-1} \sum_{t=1}^{N}\left(x_{t}(i)-f_{t}(i)\right)^{2}=\frac{1}{N-1}(\mathbf{x}(i)-\mathbf{f}(i))^{2}
$$

Observing S subjects, the variance for all subjects from their approximated models is

$$
\sigma^{2}=\sum_{i=1}^{S} \sigma_{i}^{2}
$$

Since each sample of each subject contain measurements at the same time period, we might draw these samples together in one chart as it is done in Fig. 3. 
Assuming that the deviations at each time step are differently distributed, i.e. time dependent, we might compute the variance for one time step $t$ for the set of all observed subjects to all models $i$ by

$$
\sigma_{t}^{2}=\frac{1}{S-1} \sum_{i=1}^{S}\left(x_{t}(i)-f_{t}(i)\right)^{2} \text { and } \sigma^{2}=\sum_{t=1}^{N} \sigma_{t}^{2}
$$

Now, we might analyze the system by two different approaches:

a) Either we have only one model for all subjects. Then, all samples are random deviations of the true model sample f. We select as best model the one which best "fits the data ". Biologically, the approach of only one fixed model is improbable.

b) Or we assume a different model $\mathbf{f}(i)$ for each subject $i$. This means that either the parameters of $m$ are different or even the basic model type $m$ might be different. Then, each observed sample $\mathbf{x}(\mathrm{i})$ deviate slightly from the best fitting model sample $\mathbf{f}(\mathrm{i})$. As best model $\mathrm{m}^{*}$ we might select the one which, after the individual parameter adaptation, "fits best" for all subjects.

Now, in order to evaluate the fitting of the model we have to compute the description length $\mathrm{L}$ of the data, given the model. This is the average information of the data. It can be shown that the description length $\mathrm{L}$, is bounded below by the entropy of the probability distribution of the data set $\mathrm{X}$ [7]

$$
L \geq H(X)
$$

Thus, the minimal description length of the data is obtained for the model which provides the smallest entropy of the observed data. For normally distributed data according to [8], we know that for the compound random variable $\mathrm{X}$ we have

$$
\mathrm{H}(\mathrm{X})=\ln \sqrt{(2 \pi \mathrm{e})^{\mathrm{n}} \operatorname{det} \mathrm{C}_{\mathrm{XX}}}
$$

For uncorrelated samples $\mathbf{x}$ we have

$$
\operatorname{det} C_{X X}=\prod_{t} \sigma_{t}^{2}
$$

and therefore

$$
\begin{aligned}
\mathrm{H}(\mathrm{X}, \mathrm{m}) & =1 / 2 \ln (2 \pi \mathrm{e})^{\mathrm{n}}+1 / 2 \ln \prod_{\mathrm{t}} \sigma_{\mathrm{t}}^{2}(\mathrm{~m}) \\
& =\mathrm{A}+1 / 2 \sum_{\mathrm{t}=1}^{\mathrm{n}} \ln \sigma_{\mathrm{t}}^{2}(\mathrm{~m})
\end{aligned}
$$

Therefore, the best model $\mathrm{m} *$ is the one which minimizes $\mathrm{H}(\mathrm{X}, \mathrm{m})$, i.e. which has the smallest variances for all time steps.
The information can be measured for two cases: the situation for training and the situation for testing. For training, we have the mean squared error between the observations of the training sample and those of the model sample, averaged over all variables $v$ and all models $k$ of the same type

$$
\begin{aligned}
& \mathrm{MSE}_{\text {train }}= \\
& \frac{1}{\mathrm{~S} \cdot \mathrm{m} \cdot \mathrm{N}} \sum_{\mathrm{k}=1}^{\mathrm{S}} \sum_{\mathrm{v}=1}^{\mathrm{m}} \sum_{\mathrm{t}=1}^{\mathrm{N}}\left(\mathrm{x}_{\mathrm{t}}(\mathrm{k}, \mathrm{v})-\mathrm{f}_{\mathrm{t}}(\mathrm{k}, \mathrm{v})\right)^{2}
\end{aligned}
$$

For the test case, we compare the model sample with all other possible observations, i.e. the rest of the training set, also averaged over all variables and all models of the same type

$$
\begin{aligned}
& \mathrm{MSE}_{\mathrm{est}}= \\
& \frac{1}{\mathrm{~S} \cdot \mathrm{m} \cdot \mathrm{N} \cdot(\mathrm{S}-1)} \sum_{\mathrm{k}=1}^{\mathrm{S}} \sum_{\mathrm{v}=1}^{\mathrm{m}} \sum_{\mathrm{t}=1}^{\mathrm{N}} \sum_{\mathrm{j} \neq \mathrm{k}}\left(\mathrm{x}_{\mathrm{t}}(\mathrm{j})-\mathrm{f}_{\mathrm{t}}(\mathrm{k})\right)^{2}
\end{aligned}
$$

For the two cases, the information of eq.(10) is averaged over all models $k$ of same type $\mathrm{m}$ and variables $v$ and becomes

$$
\begin{aligned}
& \mathrm{H}(\mathrm{m})=\langle\mathrm{H}(\mathrm{k}, \mathrm{v})\rangle_{\mathrm{k}, \mathrm{v}} \\
& =\frac{1}{\mathrm{~S}} \sum_{\mathrm{k}=1}^{\mathrm{S}} \frac{1}{\mathrm{~m}} \sum_{\mathrm{v}=1}^{\mathrm{m}}(1 / 2 \mathrm{~N} \cdot \ln (2 \pi \mathrm{e}) \\
& \left.+1 / 2 \sum_{\mathrm{t}=1}^{\mathrm{n}} \ln \sigma_{\mathrm{t}}^{2}(\mathrm{k}, \mathrm{v})\right)
\end{aligned}
$$

with $\sigma_{\mathrm{t}}^{2}(\mathrm{k}, \mathrm{v})=\left(\mathrm{x}_{\mathrm{t}}(\mathrm{k}, \mathrm{v})-\mathrm{f}_{\mathrm{t}}(\mathrm{k}, \mathrm{v})\right)^{2}$ for training

or

$$
\sigma_{t}^{2}(k, v)=\frac{1}{S-2} \sum_{j \neq k}^{S}\left(x_{t}(j, v)-f_{t}(k, v)\right)^{2}
$$

for testing

Therefore, we get for the averaged information $H$ of a model of type $m$

$$
\begin{aligned}
& \mathrm{H}(\mathrm{m})= \\
& \frac{1}{\mathrm{~S} \cdot \mathrm{m} \cdot \mathrm{N}} \sum_{\mathrm{k}=1}^{\mathrm{S}} \sum_{\mathrm{v}=1}^{\mathrm{m}} \sum_{\mathrm{t}=1}^{\mathrm{N}} \ln \left(\sigma_{\mathrm{t}}^{2}(\mathrm{k}, \mathrm{v})\right)+\frac{\mathrm{N}}{2} \ln (2 \pi \mathrm{e})
\end{aligned}
$$

\section{Evaluating the data simulations}

For the simulation, we generated four data files for two different model types and $M=10$ subjects. The $M$ individually generated time courses start with the same initial values, but differentiate in the following aspects:

a) All subjects have the same standard model parameters and all observations are the same. 
b) All subjects have the same standard model parameters, but the observations have random deviations $(\mathrm{N}(0,0.02)$ distribution) of the true values.

c) All subjects have individual, different model parameters $(\mathrm{N}(0,0.001)$ distribution); the observations are the true values.

d) All subjects have individual, different model parameters; but the observations have random deviations of the true values.

These four model assumptions are used to generate four observation data files. Each set of observ ations contain $n$ $=5$ sampled values of all three variables for each of the $M$ $=10$ subjects.

These four observed data files are analyzed by four different model types:

$\mathrm{m}_{1}$ ) The smaller model with $\mathrm{a}_{5}=0$.

$\mathrm{m}_{2}$ ) The "standard" model with $\mathrm{a}_{5} \neq 0$ and $\mathrm{a}_{10}, \mathrm{a}_{11}=0$.

$\mathrm{m}_{3}$ ) The augmented model with $\mathrm{a}_{5}, \mathrm{a}_{10}, \mathrm{a}_{11} \neq 0$.

$\mathrm{m}_{4}$ ) The changed (dropped and added terms) model with $\mathrm{a}_{10}, \mathrm{a}_{11} \neq 0$ and $\mathrm{a}_{5}=0$.

Each of the four observation files is used to adapt the parameters of each of the $\mathrm{M}=10$ subjects of the same model type to the observations by the evolutionary method described in section 3 . So, we get 16 result sets for 10 subjects each.

For each adaptation try we use 100 cycles of adapting all parameters in order to minimize the mean squared error $\mathrm{R}$ between the model prediction and the observations. For each subject, 10 tries are performed and the one with the smallest $\mathrm{R}$ is recorded in order to avoid getting stuck in a suboptimum. After adaptation, the performance of the models was evaluated by computing the minimum description length, i.e. entropy $\mathrm{H}$ for the model adaptations. The evaluated values for the entropy $\mathrm{H}$ for the four model types $m_{1}, m_{2}, m_{3}, m_{4}$ adapting to the data of the four observed situations a),b),c) and d) are presented in table 1 .

\begin{tabular}{c|cc|cc} 
& $\mathrm{H}_{\mathrm{a}}$ & $\mathrm{H}_{\mathrm{b}}$ & $\mathrm{H}_{\mathrm{c}}$ & $\mathrm{H}_{\mathrm{d}}$ \\
\hline $\mathrm{m}_{1}$ & $-90,34$ & $-61,07$ & $-86,34$ & $-79,73$ \\
$\mathbf{m}_{2}$ & $\mathbf{- 1 0 5 , 8 9}$ & $\mathbf{- 1 0 5 , 1 1}$ & $\mathbf{- 1 2 3 , 8 3}$ & $\mathbf{- 8 1 , 0 3}$ \\
$\mathrm{m}_{3}$ & $-63,90$ & $-67,32$ & $-76,43$ & $-33,87$ \\
$\mathrm{~m}_{4}$ & $-53,70$ & $-82,50$ & $-110,60$ & $-58,06$ \\
\hline
\end{tabular}

\section{Table 1 The evaluated observations for $n=5$ samples}

What can we conclude by these results?

Keeping in mind that $\mathrm{m}_{2}$ is the standard model type that was used to produce all data, we see that this model type has the smallest entropy of all other models - it turns out as the best model to select. Therefore, our model selection criterion is valid in our example.
For the case d) of different parameter regimes and random deviations the smaller model fits only slightly worse to the data. Why? The reason behind is that the random deviations and the systematic deviations are in the same range; for only a small number of observations for one individual $(n=5)$ the difference becomes hard to detect.

Here we encounter a fundamental problem of data modeling: how do we know that for a given observation variance the number of observed data points are sufficient to select a model properly? What difference of complexity should be taken as a reason for a model to be more valid than another one? Theses questions are still open for research.

\section{Discussion}

Data driven modeling is an important attempt to rationalize the efforts of creating models guided not by as sumptions but by reality. The paper shows some of the problems involved in this kind of modeling and proposes the minimum description length of the observed data as selection criterion.

For the small but important problem of inflammation and septic shock differential equations we consider four different models types: a standard model, the model with one term dropped, the model with two additional terms and a changed model. These four models are confronted with synthetic data, generated by random versions of the standard model. Here, all four possible model types converge more or less fast to fit the data; no terms can be pruned due to small parameter values; an automatic tailoring of the model to the data is not possible.

Thus, the model selection can neither be based on the convergence speed nor on the "complexity" of the formulas (is a multiplication more complex than an addition?) but have to be based on another criterion. In this paper we chose the minimum description length MDL of the data using the model as performance criterion. Assuming normally distributed deviations we computed the entropy as lower limit of the MDL by using the observed variance between the adapted models and the observed data. The simulation results validated our approach: The analyzing model describes the data with the lowest MDL if data generation model type and analyzing model type coincide.

\section{References}

[1] R. Brause, E.Hanisch, J.Paetz, B. Arlt: The MEDAN project: results and practical meanings, 3. Int. Symp osium "Sepsis, SIRS, Immune Response - Concepts, Diagnostics and Therapy", A. Nierhaus, J. Schulte am Esch (Eds.), PABST Science Publishers, Lengerich, Germany, (2003), pp.122-129

[2] Steffen, M., Petti, A., D'haeseleer, P., Aach, J., and Church, G.M. (2002) Automated Modeling of Signal 
Transduction Networks. BMC Bioinformatics 3:3444.

[3] C. Chow: Development of a Dynamical Systems Model of Acute Inflammation, E. Neugebauer: $2^{\text {nd }}$ workshop on complex systems: Analysis in Shock and Trauma Research, University of Cologne, (2003)

[4] R. Brause: Adaptive modeling of biochemical pathways. IEEE 15th Int. Conf on Tools with Art. Intell. ICTAI-2003, IEEE Press 2003, pp.62-68, (2003)

[5] H.-P. Schwefel: Evolution and Optimum Seeking , J.Wiley, (1995)

[6] I. N. Bronstein et al., Handbook of Mathematics, Van Nostrand Reinhold; 3rd edition (1991)

[7] M. Hansen, B. Yu: Model selection and the principle of Minimum Description Length, Journal of the American Statistical Association, Vol.96 (454),pp. 746-774, 2001

[8] T.Cover, J.Thomas: Elements of Information Theory, John Wiley, New York 1991 